\title{
Noncommutative Wilson lines in higher-spin theory and correlators of free conformal fields
}

\author{
Roberto Bonezzi \\ Groupe de Mécanique et Gravitation, UMONS; 20 place du Parc, 7000 Mons, Belgium \\ E-mail: roberto.bonezzi@umons.ac.be

\section{Nicolas Boulanger} \\ Groupe de Mécanique et Gravitation, UMONS; 20 place du Parc, 7000 Mons, Belgium \\ E-mail: nicolas.boulanger@umons.ac.be
}

\section{David De Filippi*}

Groupe de Mécanique et Gravitation, UMONS; 20 place du Parc, 7000 Mons, Belgium

E-mail: david.defilippieumons.ac.be

\section{Per Sundell}

Departamento de Ciencias Físicas, Universidad Andres Bello; Republica 220, Santiago de Chile

E-mail: per.anders.sundell@gmail.com

\begin{abstract}
In this note, we review the results of 1705.03928 on a special class of observables of Vasiliev's four-dimensional higher spin gravity theory, referred to as zero-form charges. These objects were shown to be twisted open Wilson lines in the noncommutative twistor $\mathrm{Z}$ space. For boundary conditions corresponding to higher spin fields in asymptically anti-de Sitter spacetime, their classical perturbative expansion was then proven to start with cyclically-invariant building blocks of n-point correlation functions of bilinear operators in free conformal field theories (CFT) in three dimensions. On the CFT side, the proof involves the explicit computation of the aforementioned building blocks in arbitrary dimension $\mathrm{d}>2$, which generalizes known results on 3-point functions for any $\mathrm{d}>2$, and on $\mathrm{n}$-point functions for $\mathrm{d}=3$.
\end{abstract}

Corfu Summer Institute 2017 "School and Workshops on Elementary Particle Physics and Gravity" 2-28 September 2017

Corfu, Greece

${ }^{*}$ Speaker. 


\section{Introduction}

Despite many no-go theorems restricting the interactions among higher-spin fields (see [1] for a review), Vasiliev was able [2] to write fully non-linear equations describing an infinite tower of Fronsdal fields [3], with unbounded integer spins, propagating around the $A d S_{4}$ spacetime and interacting non-trivially. The interactions come via the introduction of a four-dimensional noncommutative extension of spacetime, called $Z$-space.

Later on, the theory was conjectured to be holographically dual to different $U(N)$ vector models on the three-dimensional conformal boundary, according to the values taken by a free dimensionless parameter of the theory and to the choice of boundary conditions for the scalar field propagator $^{1}$. More precisely, two specific values of the parameter yield parity invariant models : on the one hand the type A model that was conjectured $[4,5]$ to be dual to free and critical models of bosons and on the other hand the type B model that was conjectured [6, 7] to be dual to free and critical models of fermions. All the other values give a one-parameter family of parity breaking higher-spin models that were conjectured $[8,9]$ to be dual to Chern-Simons matter theories.

In [10], on which this work is based, we focussed on the simplest form of this duality, first spelled out in [4], that relates Vasiliev's type A model, via propagators obeying the irregular condition, to the free bosonic $U(N)$ vector model. It has the particular feature of being a holographic duality linking two weakly coupled theories. Different verifications of the duality gave some positive results at cubic and quartic order [11, 12]. However, the authors of [12] found divergences for some couplings, which afterwards were shown [13] to be related to nonlocality of the interaction vertices on $A d S_{4}$ from Vasiliev's equations. Although there has been a lot of progress (see e.g. $[14,15,16])$, no completely satisfactory solution to this problem exists so far.

Instead of asking the vertices of the field equations to be defined as local quantities in $A d S_{4}$, it was proposed in [17] that locality be studied in terms of quantities possessing the property of being pertubatively additive for two well-separated one-body solutions. The proposal of the authors of [18] was then to study locality through the evaluation of some gauge invariant objects called zeroform charges $[19,20]$, that are thought to exhibit this cluster-decomposition property, and that were effectively shown to have it for a black-hole-like solution [21, 22, 23].

The idea of this program is to compute bulk quasi-amplitudes by evaluating zero-form charges and in some sense to plug to it the bulk-to-boundary propagators constructed in [12]. This was shown [24] to reproduce the boundary three-point functions at leading and first subleading order in weak field expansion. The leading $n$-point quasi-amplitudes were then computed $[25,26]$ using a modification of the zero-form charges which is specific to the leading order and found agreement with the boundary results of [27], where an explicit expression was given for $n$-point functions in the $C F T_{d}$ for $d=3$.

In [10], we first showed that the zero-form charges could be obtained from a noncommutative Yang-Mills perspective as decorated Wilson lines in Z-space. We reproduced the result of [25] with a method amenable to the corrections induced by $Z$-space. We computed the $n$-point functions of conserved currents in the free $C F T_{d}$ by explicitly using Wick theorem, and in the three-dimensional case we found agreement with the zero-form charges, already at the level of cyclic structures.

\footnotetext{
${ }^{1}$ Though the propagators of the spin $s \geq 1$ must obey the Dirichlet boundary condition, the one for the scalar field can have either the Dirichlet (or "regular") boundary condition or the Neumann (or "irregular") one.
} 
In this note we outline the major results of [10], while referring to that paper for technical details. The paper is organized as follows. In Section 2 the very basics of the considered model and related notations are introduced. In Section 3, we point out the analogy with non-commutative gauge theories, and we define zero-form charges, quasi-amplitudes and pre-amplitudes in terms of open Wilson lines. In Section 4 we evaluate the pre-amplitudes on the bulk-to-boundary propagators on one side, the $n$-point correlators in the free $U(N)$ model on the other side and we discuss their correspondence.

\section{Vasiliev's bosonic model}

Four-dimensional bosonic Vasiliev's higher spin theories are formulated on a $\mathscr{Y}_{4}$ bundle over a base manifold $\mathscr{X}_{4} \times \mathscr{Z}_{4}$. In the notations that we shall use, $x^{\mu}$ are the four coordinates of the usual spacetime $\mathscr{X}_{4}, Z^{\underline{\alpha}}=\left(z^{\alpha},-\bar{z}^{\dot{\alpha}}\right)$ are the coordinate of its four-dimensional noncommutative extension $\mathscr{Z}_{4}$ and $Y^{\underline{\alpha}}=\left(y^{\alpha}, \bar{y}^{\dot{\alpha}}\right)$ are the coordinates of the four-dimensional fiber $\mathscr{Y}_{4}$. The twelvedimensional correspondence space $\mathscr{X}_{4} \times \mathscr{Z}_{4} \times \mathscr{Y}_{4}$ is equipped with a product, denoted by $\star$, ensuring the following commutation relations

$$
\left[Y^{\underline{\alpha}}, Y^{\underline{\beta}}\right]_{\star}=2 i C^{\underline{\alpha} \underline{\beta}}, \quad\left[Z^{\underline{\alpha}}, Z^{\underline{\beta}}\right]_{\star}=-2 i C^{\underline{\alpha} \underline{\beta}}, \quad\left[Y^{\underline{\alpha}}, Z^{\underline{\beta}}\right]_{\star}=0,
$$

where $C^{\underline{\alpha} \underline{\beta}}$ is an $S p(4)$ invariant non-degenerate tensor. $C_{\underline{\alpha} \underline{\beta}}$ can be expressed in terms of the $\operatorname{Sl}(2)$ invariant tensors ${ }^{2}$ as

$$
C^{\underline{\alpha} \underline{\beta}}=\left(\begin{array}{cc}
\varepsilon^{\alpha \beta} & 0 \\
0 & \varepsilon^{\dot{\alpha} \dot{\beta}}
\end{array}\right), \quad C_{\underline{\alpha} \underline{\beta}}=\left(\begin{array}{cc}
\varepsilon_{\alpha \beta} & 0 \\
0 & \varepsilon_{\dot{\alpha} \dot{\beta}}
\end{array}\right) .
$$

In the rest of the paper we use the NW-SE convention ${ }^{3}$ and omit most of the spinorial indices.

The fields are (locally defined) horizontal differential forms on the total space $\mathscr{X}_{4} \times \mathscr{Z}_{4} \times \mathscr{Y}_{4}$. By horizontal, we mean that we never use $\mathrm{d} Y \underline{\alpha}$, i.e. that the relevant differential calculus is based on the following horizontal differential:

$$
\widehat{\mathrm{d}}:=\mathrm{d}+\mathrm{q}, \quad \mathrm{d}:=\mathrm{d} x^{\mu} \partial_{\mu}^{x}, \quad \mathrm{q}:=\mathrm{d} Z^{\underline{\alpha}} \partial_{\underline{\alpha}}^{Z},
$$

which obeys the graded Leibniz rule

$$
\widehat{\mathrm{d}}(\hat{f} \star \hat{g})=(\widehat{\mathrm{d}} \hat{f}) \star \hat{g}+(-)^{\operatorname{deg} \hat{f} \operatorname{deg} \hat{g}} \hat{f} \star(\widehat{\mathrm{d}} \hat{g}),
$$

where $\hat{f}$ and $\hat{g}$ are forms ${ }^{4}$, deg denotes the total form degree and the exterior product $\wedge$ is left implicit.

Instead of treating $Z^{\underline{\alpha}}$ and $Y^{\underline{\alpha}}$ as noncommuting operators, we shall see them as ordinary complex variables by introducing a commutative pointwise product. The $\star$-product is then defined

\footnotetext{
${ }^{2} \varepsilon_{\alpha \beta}$ and $\varepsilon_{\dot{\alpha} \dot{\beta}}$ are defined by the fact that they are antisymmetric and by the convention $\varepsilon_{12}=\varepsilon^{12}=\varepsilon_{1 \dot{2}}=\varepsilon^{\mathrm{i} \dot{2}}=1$

${ }^{3} S p(4)$ indices are raised as $V \underline{\alpha}:=C \underline{\alpha} \underline{\beta} V_{\beta}$, lowered as $V_{\underline{\alpha}}:=V \underline{\beta} C_{\beta} \underline{\alpha}$ and contracted as: $U V:=U \underline{\alpha} V_{\underline{\alpha}}$. $\varepsilon_{\alpha \beta}$ and $\varepsilon_{\dot{\alpha} \dot{\beta}}$ are used in the same fashion for $\overline{S l}(2)$ indices.

${ }^{4} \mathrm{We}$ put hats on objects that are nontrivial differential forms on $\mathscr{Z}_{4}$, including zero-forms with nontrivial $Z$ dependence.
} 
in terms of this pointwise product via the following twisted convolution formula

$$
(\hat{f} \star \hat{g})(x, Z ; Y ; \mathrm{d} x, \mathrm{~d} Z):=\int \frac{d^{4} U d^{4} V}{(2 \pi)^{4}} e^{i \underline{\underline{\alpha}}^{\underline{\alpha}}} \underline{\underline{\alpha}} \hat{f}(x, Z+U ; Y+U ; \mathrm{d} x, \mathrm{~d} Z) \hat{g}(x, Z-V ; Y+V ; \mathrm{d} x, \mathrm{~d} Z),
$$

for auxiliary variables $U^{\underline{\alpha}}:=\left(u^{\alpha}, \bar{u}^{\dot{\alpha}}\right)$ and $V^{\underline{\alpha}}:=\left(v^{\alpha}, \bar{v}^{\dot{\alpha}}\right)$. The set of bounded functions of $Y$ and $Z$ whose complex modulus is integrable is closed under $\star$-product and admits a trace operation, given by

$$
\operatorname{Tr} \hat{f}(Z, Y):=\int \mathrm{d}^{4} Z \mathrm{~d}^{4} Y \hat{f}(Z, Y)
$$

which makes cyclic the $\star$-products inside of it, in the following sense:

$$
\operatorname{Tr} \hat{f} \star \hat{g}=\operatorname{Tr} \hat{g} \star \hat{f} .
$$

The field variables of the theory are a zero-form field $\widehat{\Phi}(x, Z ; Y)$ and a one-form gauge connection $\widehat{A}=\mathrm{d} x^{\mu} \widehat{A}_{\mu}(x, Z ; Y)+\mathrm{d} Z \underline{\alpha}^{\alpha} \widehat{A}_{\underline{\alpha}}(x, Z ; Y)$. They are associated with a transformation law given in terms of a $\star$-invertible gauge function $\widehat{g}(x, Z ; Y)$ by

$$
\widehat{A} \longrightarrow \widehat{g} \star \widehat{\mathrm{d}} \widehat{g}^{-1}+\widehat{g} \star \widehat{A} \star \widehat{g}^{-1}, \quad \widehat{\Phi} \longrightarrow \widehat{g} \star \widehat{\Phi} \star \pi\left(\widehat{g}^{-1}\right) .
$$

More specifically, the variables in Vasiliev's bosonic models are $\widehat{A}$ and $\widehat{\Phi}$ submitted to two kinematical constraints. Accordingly, the gauge functions will be the ones preserving them. The first constraint is the reality condition

$$
\widehat{A}^{\dagger}=-\widehat{A}, \quad \widehat{\Phi}^{\dagger}=\pi(\widehat{\Phi}), \quad \widehat{g}^{\dagger}=\widehat{g}^{-1}
$$

where

$$
\begin{array}{r}
(x, y, \bar{y}, z,-\bar{z})^{\dagger}=(x, \bar{y}, y, \bar{z},-z), \\
(\widehat{\mathrm{d}} \hat{f})^{\dagger}=\widehat{\mathrm{d}} \hat{f}^{\dagger}, \quad(\hat{f} \star \hat{g})^{\dagger}=(-)^{\operatorname{deg} \hat{f} \operatorname{deg} \hat{g}} \hat{g}^{\dagger} \star \hat{f}^{\dagger},
\end{array}
$$

for every pair of differential forms $\hat{f}$ and $\hat{g}$. The second one is the bosonic projection

$$
\pi(\widehat{A})=\bar{\pi}(\widehat{A}), \quad \widehat{\Phi}^{\dagger}=\pi(\widehat{\Phi})=\bar{\pi}(\widehat{\Phi}), \quad \pi(\widehat{g})=\bar{\pi}(\widehat{g})
$$

where

$$
\begin{array}{cl}
\pi(x, y, \bar{y}, z,-\bar{z})=(x,-y, \bar{y},-z,-\bar{z}), & \bar{\pi}(x, y, \bar{y}, z,-\bar{z})=(x, y,-\bar{y}, z, \bar{z}), \\
\pi(\widehat{\mathrm{d}} \hat{f})=\widehat{\mathrm{d}} \pi(\hat{f}), \pi(\hat{f} \star \hat{g})=\pi(\hat{f}) \star \pi(\hat{g}), & \bar{\pi}(\widehat{\mathrm{d}} \hat{f})=\widehat{\mathrm{d}} \bar{\pi}(\hat{f}), \bar{\pi}(\hat{f} \star \hat{g})=\bar{\pi}(\hat{f}) \star \bar{\pi}(\hat{g}) .
\end{array}
$$

The purpose of the latter is to select, among the fields living on $\mathscr{X}_{4}$, the ones that have integer spin. It is possible to further constrain them and select only the even spin part, thereby defining the minimal bosonic models, in the following way:

$$
\tau(\widehat{A})=-\widehat{A}, \quad \tau(\widehat{\Phi})=\pi(\widehat{\Phi}), \quad \tau(\widehat{g})=\widehat{g}^{-1}
$$


where

$$
\begin{array}{r}
\tau(x, y, \bar{y}, z,-\bar{z})=(x, i y, i \bar{y},-i z, i \bar{z}), \\
\tau(\widehat{\mathrm{d}} \hat{f})=\widehat{\mathrm{d}} \tau(\hat{f}), \quad \tau(\hat{f} \star \hat{g})=(-)^{\operatorname{deg} \hat{f} \operatorname{deg} \hat{g}} \tau(\hat{g}) \star \tau(\hat{f}) .
\end{array}
$$

For the purpose of computing observables, we are interested in mapping the master fields $\widehat{A}$ and $\widehat{\Phi}$ to operators $\widehat{f}(x, Z ; Y ; \mathrm{d} x, \mathrm{~d} Z)$ transforming in the adjoint representation of the higherspin algebra, i.e. transforming under (2.8) as $\widehat{f} \longrightarrow \widehat{g} \star \widehat{f} \star \widehat{g}^{-1}$. Since $\widehat{U}:=\mathrm{d} x^{\mu} \widehat{A}_{\mu}$ is a gauge connexion on a commutative space ${ }^{5}$, it is known that its gauge covariant information is contained in its curvature $\widehat{\mathrm{d}} \widehat{U}+\widehat{U} \star \widehat{U}$. Conversely, its noncommutative counterpart $\widehat{A}_{\underline{\alpha}}$ can be univoquely associated with the following adjoint quantity:

$$
\widehat{S}_{\underline{\alpha}}:=Z_{\underline{\alpha}}-2 i \widehat{A}_{\underline{\alpha}},
$$

usually called deformed oscillator ${ }^{6}$. In the case of $\widehat{\Phi}$, the twisted adjoint representation can be "untwisted" by means of the following remarkable representation of the automorphisms $\pi$ and $\bar{\pi}$ on a zero-form $\hat{f}_{[0]}$ :

$$
\pi\left(\hat{f}_{[0]}\right)=\hat{\kappa} \star \hat{f}_{[0]} \star \hat{\kappa}, \quad \bar{\pi}\left(\hat{f}_{[0]}\right)=\hat{\bar{\kappa}} \star \hat{f}_{[0]} \star \hat{\bar{\kappa}},
$$

in terms of the so-called Klein operators

$$
\hat{\kappa}:=e^{i y^{\alpha} z_{\alpha}}, \quad \hat{\bar{\kappa}}:=e^{-i \bar{y}^{\dot{\alpha}} \bar{z} \alpha},
$$

that enjoy the additional properties

$$
\hat{\kappa}^{\dagger}=\hat{\bar{\kappa}}, \quad \hat{\kappa} \star \hat{\kappa}=1=\hat{\bar{\kappa}} \star \hat{\bar{\kappa}}, \quad \hat{\kappa} \star \hat{\bar{\kappa}}=\hat{\bar{\kappa}} \star \hat{\kappa} .
$$

This allows to define two adjoint quantities

$$
\widehat{\Psi}:=\widehat{\Phi} \star \hat{\kappa}, \quad \widehat{\bar{\Psi}}:=\widehat{\Phi} \star \hat{\bar{\kappa}} .
$$

The field equations, called Vasiliev's equations, can be written [2] in terms of these adjoint variables as

$$
\begin{aligned}
& \mathrm{d} \widehat{U}+\widehat{U} \star \widehat{U}=0, \\
& {\left[\widehat{S}_{\alpha}, \widehat{S}_{\dot{\alpha}}\right]_{\star}=0,} \\
& \mathrm{~d} \widehat{S}_{\alpha}+\left[\widehat{U}, \widehat{S}_{\alpha}\right]_{\star}=0, \\
& \mathrm{~d} \widehat{S}_{\dot{\alpha}}+\left[\widehat{U}, \widehat{S}_{\dot{\alpha}}\right]_{\star}=0, \\
& \mathrm{~d} \widehat{\Psi}+[\widehat{U}, \widehat{\Psi}]_{\star}=0, \\
& \mathrm{~d} \widehat{\bar{\Psi}}+[\widehat{U}, \widehat{\bar{\Psi}}]_{\star}=0, \\
& \left\{\widehat{S}_{\alpha}, \widehat{\Psi}\right\}_{\star}=\left[\widehat{S}_{\alpha}, \widehat{\bar{\Psi}}\right]_{\star}=0, \\
& {\left[\widehat{S}_{\dot{\alpha}}, \widehat{\Psi}\right]_{\star}=\left\{\widehat{S}_{\dot{\alpha}}, \widehat{\Psi}\right\}_{\star}=0,} \\
& {\left[\widehat{S}_{\alpha}, \widehat{S}_{\beta}\right]_{\star}+2 i \varepsilon_{\alpha \beta}\left(1-e^{i \theta} \widehat{\Psi}\right)=0 \text {, }} \\
& {\left[\widehat{S}_{\dot{\alpha}}, \widehat{S}_{\dot{\beta}}\right]_{\star}+2 i \varepsilon_{\dot{\alpha} \dot{\beta}}\left(1-e^{-i \theta} \widehat{\bar{\Psi}}\right)=0 \text {, }}
\end{aligned}
$$

\footnotetext{
${ }^{5}$ This can be understood if, for a moment, the total space is interpreted as a $\mathscr{Z}_{4} \times \mathscr{Y}_{4}$ bundle over $\mathscr{X}_{4}$ and the index $\underline{\alpha}$ in $\widehat{A}_{\underline{\alpha}}$ is seen as internal.

${ }^{6}$ Indeed, (2.27) can be viewed [28] as a generalized version of Wigner's deformation [29, 30] of the Heisenberg algebra.
} 
in terms of a real constant $\theta \in[0,2 \pi]$ whose value determines the model. We are interested in two specific values yielding the parity-invariant models [7]: $\theta=0$ for the Type A model and $\theta=\frac{\pi}{2}$ for the Type B model. The equations are compatible with the gauge transformation (2.8) and with the constraints $(2.9,2.12,2.15)$.

The vacuum solution describing the $A d S_{4}$ background is obtained by setting $\widehat{\Phi}^{(0)}=0, \widehat{S}_{\underline{\alpha}}^{(0)}=$ $Z_{\underline{\alpha}}$ and taking $\widehat{U}^{(0)}$ as the Cartan connection of $A d S_{4}$, given by

$$
\Omega(Y \mid x)=\frac{1}{4 i}\left(y^{\alpha} y^{\beta} \omega_{\alpha \beta}+\bar{y}^{\dot{\alpha}} \bar{y}^{\dot{\beta}} \bar{\omega}_{\dot{\alpha} \dot{\beta}}+2 y^{\alpha} \bar{y}^{\dot{\alpha}} h_{\alpha \dot{\alpha}}\right) .
$$

One may then perform a perturbative expansion around this background and find, at the linearised level the free propagation of an infinite tower of Fronsdal fields around $A d S_{4}$. In particular, $\widehat{\Psi}^{(1)}$ is given by $\Phi \star \hat{\kappa}$, where $\Phi(x, Y)$ is a covariantly constant $Z$-independent zero-form.

\section{Observables in Vasiliev's theory}

The purpose of this section is to use analogies that exist between Vasiliev's theory and noncommutative Yang-Mills theory (for the latter formalism, see e.g. [31, 32, 33]) to build functionals that are higher spin gauge as well as diffeomorphism invariant, hence serving as candidates for observables. Indeed, since $\widehat{A}$ transforms like a Yang-Mills connection, one can construct gauge invariants from Wilson lines associated with curves

$$
\mathscr{C}:[0,1] \rightarrow \mathscr{X}_{4} \times \mathscr{Z}_{4}: \sigma \rightarrow\left(\xi^{\mu}(\sigma), \xi^{\underline{\alpha}}(\sigma)\right)
$$

that are closed in $\mathscr{X}_{4}$ and open in $\mathscr{Z}_{4}$, i.e.

$$
\xi^{\mu}(0)=\xi^{\mu}(1)=0, \quad \xi^{\underline{\alpha}}(0)=0, \quad \xi^{\underline{\alpha}}(1)=2 M^{\underline{\alpha}}=2 C^{\underline{\alpha}} \underline{\underline{\beta}} M_{\underline{\beta}} .
$$

The associated Wilson line is defined as

$$
\begin{aligned}
W_{\mathscr{C}}(x, Z ; Y): & =\operatorname{Pexp}_{\star}\left(\int_{0}^{1} \mathrm{~d} \sigma\left(\dot{\xi}^{\mu}(\sigma) \widehat{A}_{\mu}(\sigma)+\dot{\xi} \underline{\alpha}(\sigma) \widehat{A}_{\underline{\alpha}}(\sigma)\right)\right) \\
& =\sum_{n=0}^{\infty} \int_{0}^{1} \mathrm{~d} \sigma_{n} \ldots \int_{0}^{\sigma_{2}} \mathrm{~d} \sigma_{1} \underset{i=1}{\star}\left(\dot{\xi}^{\mu}\left(\sigma_{i}\right) \widehat{A}_{\mu}\left(\sigma_{i}\right)+\dot{\xi} \underline{\alpha}\left(\sigma_{i}\right) \widehat{A}_{\underline{\alpha}}\left(\sigma_{i}\right)\right),
\end{aligned}
$$

where $\widehat{A}(\sigma):=\widehat{A}\left(x^{\mu}+\xi^{\mu}(\sigma), Z^{\underline{\alpha}}+\xi \underline{\alpha}(\sigma) ; Y^{\underline{\alpha}}\right)$ and for any set of $n$ functions $\left\{\hat{f}_{1}, \ldots, \hat{f}_{n}\right\}$, the symbol $\star_{i=1}^{n} \hat{f}_{i}$ is defined as $\hat{f}_{1} \star \ldots \star \hat{f}_{n}$ in that order. The gauge transformation (2.8) acts on it as

$$
W_{\mathscr{C}}(x, Z ; Y) \longrightarrow \widehat{g}(x, Z ; Y) \star W_{\mathscr{C}}(x, Z ; Y) \star \widehat{g}^{-1}(x, Z+2 M ; Y) .
$$

Unlike the traces of open Wilson lines in commutative spaces, their counterparts in a noncommutative space ${ }^{7}$ can be made gauge invariant by inserting the translation operator $e^{i M Z}$, characterized by the following property :

$$
\hat{f}(x, Z+2 M ; Y) \star e^{i M Z}=e^{i M Z} \star \hat{f}(x, Z ; Y),
$$

\footnotetext{
${ }^{7}$ Although in that case the trace includes an integration over the non-commutative space.
} 
at the end of the Wilson line. Thus, for any curve $\mathscr{C}$ satisfying (3.2) and for any operator $\hat{O}(x, Z ; Y)$ transforming as $\hat{O} \longrightarrow \widehat{g} \star \hat{O} \star \widehat{g}^{-1}$, the quantity

$$
\widetilde{O}_{\mathscr{C}}(M \mid x):=\operatorname{Tr}\left[\hat{O}(x, Z ; Y) \star W_{\mathscr{C}}(x, Z ; Y) \star e^{i M Z}\right],
$$

is gauge invariant. As argued in [31], the consideration of a generic adjoint impurity $\hat{O}$ allows one to only study observables associated with the straight line

$$
L_{2 M}:[0,1] \rightarrow \mathscr{X}_{4} \times \mathscr{Z}_{4}: \sigma \rightarrow\left(0,2 \sigma M^{\underline{\alpha}}\right) .
$$

In that case, we showed the following identity

$$
\exp _{\star}(i M \widehat{S})=W_{L_{2 M}}(x, Z ; Y) \star \exp (i M Z),
$$

allowing to rewrite the observables as a deformed Fourier transform, viz.

$$
\widetilde{O}_{L_{2 M}}(M \mid x)=\int \mathrm{d}^{4} Z \mathrm{~d}^{4} Y \hat{O}(x, Z ; Y) \star \exp _{\star}(i M \widehat{S}),
$$

which exhibits the role of $M^{\underline{\alpha}}$ as a twistor space momentum variable.

From the field equations $(2.23-2.27)$, it follows that the space of adjoint operators that can be built out of the master fields on-shell is spanned by

$$
\hat{O}_{n_{0}, t ; \underline{\alpha}_{1}, \cdots, \underline{\alpha}_{K}}:=\widehat{\Psi}^{\star n_{0}} \star(\hat{\kappa} \hat{\bar{\kappa}})^{\star t} \star \widehat{S}_{\left(\underline{\alpha}_{1}\right.} \star \cdots \star \widehat{S}_{\left.\underline{\alpha}_{K}\right)} .
$$

As a consequence, the space of twisted open Wilson lines, thought of as a linear space of observables, admits the following basis

$$
\mathscr{I}_{n_{0}, t}(M)=\int \mathrm{d}^{4} Z \mathrm{~d}^{4} Y \widehat{\Psi}^{\star n_{0}} \star(\hat{\kappa} \hat{\bar{\kappa}})^{\star t} \star \exp _{\star}(i M \widehat{S}),
$$

indicized by $\left(n_{0}, t ; M\right)$. In particular, the insertions of $\widehat{S}_{\underline{\alpha}}$ are reproduced by taking derivatives with respect to $M^{\underline{\alpha}}$. The observables (3.11) have been considered and evaluated in some special cases in $[18,34,24]$, where they were referred to as zero-form charges.

In the weak field expansion scheme, we can write their leading order contribution as

$$
\mathscr{I}_{n_{0}, t}^{\left(n_{0}\right)}(M)=\int \mathrm{d}^{4} Z \mathrm{~d}^{4} Y\left(\underset{i=1}{\stackrel{n_{0}}{\star}} \Phi\left(x ;(-)^{i+1} y, \bar{y}\right)\right) \star e^{i e y z} \star e^{-i t \bar{y} \bar{z}} \star e^{i \mu z} \star e^{-i \bar{\mu} \bar{z}},
$$

upon decomposing $M_{\underline{\alpha}}=\left(\mu_{\alpha}, \bar{\mu}_{\dot{\alpha}}\right)$, using (2.19) and defining

$$
e:=n_{0}+t \quad \bmod 2, \quad t \in\{0,1\} .
$$

Thinking of the zero-form charges as contributions to a free-energy functional ${ }^{8}$, we are interested in pre-amplitudes

$$
\mathscr{A}_{n_{0}, t}\left(\Phi_{i} \mid M\right):=\int \mathrm{d}^{4} Z \mathrm{~d}^{4} Y\left(\stackrel{n_{0}}{\star} \underset{i=1}{\star} \Phi_{i}\left(x ;(-)^{i+1} y, \bar{y}\right)\right) \star e^{i e y z} \star e^{-i t \bar{y} \bar{z}} \star e^{i \mu z} \star e^{-i \bar{\mu} \bar{z}},
$$

\footnotetext{
${ }^{8}$ One can argue that the zero-form charges are extensive observables in the sense that they are additive for systems in asymptoticaly anti-de Sitter geometries consisting of spatially separated sub-systems [21].
} 
where the quantities $\Phi_{i}, i=1, \ldots, n$ are $n$ different fields transforming as $\Phi$, all with the same gauge parameter. As the pre-amplitudes are distributions, the actual finite observables are given by

$$
\mathscr{A}_{n_{0}, t}^{\mathscr{V}}\left(\Phi_{i}\right):=\int \mathrm{d}^{4} M \widetilde{\mathscr{V}}(M) \mathscr{A}_{n_{0}, t}\left(\Phi_{i} \mid M\right)
$$

for bounded and integrable smearing functions $\widetilde{\mathscr{V}}(M)$. Thus, the information contained in the $M$ dependence of the pre-amplitudes can be obtained by using derivatives of a basic smearing function that is real-analytic at $M=0$ and that has finite moments in $M$-space. To this end, one can show that the dependence on $\widetilde{\mathcal{V}}(M)$ only appears through an overall normalization, viz.

$$
\mathscr{A}_{n_{0}, t}^{\mathscr{V}}\left(\Phi_{i}\right)=\widetilde{\mathscr{V}}_{t, e} \mathscr{A}_{n_{0}, t}^{\mathscr{V}}\left(\Phi_{i}\right)
$$

where $\widetilde{\mathscr{V}}_{t, e}(t, e \in\{0,1\})$ are given in terms of the value of the smearing function at $M=0$ and its first moment.

The complete symmetrization of the pre-amplitudes under the exchange of the different legs $\Phi_{i}$ give the quasi-amplitudes studied in [24]. As shown in [24, 25], at the leading order, i.e. $n=n_{0}$, these reproduce the correlation functions of bilinear operators in the free conformal field theory in three dimensions for $n=2,3$ and 4 .

One can show that the pre-amplitudes are invariant under cyclic permutations of the $\Phi_{i}$, raising the question of whether there exists some corresponding cyclic structure in the dual CFT.

\section{Zero-form charges and holography}

In this section, we review the evaluation of the pre-amplitudes (3.14) with $\Phi_{i}$ given by the Weyl zero-form $\mathscr{K}_{i}$ of a tower of Fronsdal fields propagated from a point $x_{i}$ on the conformal boundary to a common bulk point $x_{0}[11,12]$, including a bulk scalar field obeying the Neumann boundary condition. We then spell out the computation of the cyclic building blocks for the $n$-point correlation functions of conserved currents in the free $U(N)$ vector model in any dimension $d$, and find agreement with the pre-amplitudes of Vasiliev's non-minimal Type A model in the case of $d=3$. Finally, we discuss the case of the analogous correspondence between the minimal bosonic Type A model and the free $O(N)$ vector model in $d=3$.

In terms of a boundary polarization spinor ${ }^{9} \chi_{i}$ the aforementioned Weyl zero-form reads [24]

$$
\mathscr{K}_{i}\left(x_{0}, x_{i}, \chi_{i} \mid Y\right):=K_{i} e^{i y \Sigma_{i} \bar{y}} \sum_{\sigma_{i}= \pm 1}\left(e^{i \theta} e^{i \sigma_{i} \bar{v}_{i} \bar{\Sigma}_{i} y}+e^{-i \theta} e^{i \sigma_{i} v_{i} \Sigma_{i} \bar{y}}\right)
$$

Using Poincaré coordinates $x^{\mu}=(r, \vec{x})$, where $r$ vanishes at the conformal boundary ${ }^{10}$, for $A d S_{4}$

\footnotetext{
${ }^{9} \mathrm{~A}$ spinor on the conformal boundary of $A d S_{4}$, which has 2 independent components, can be written as a 4dimensional Dirac spinor submitted to a reality condition. For the polarization spinor, the condition is $\left(\chi_{i}\right)^{\dagger}=\bar{\chi}_{i}=\bar{\sigma}^{r} \chi_{i}$, in terms of the coordinate $r$ that vanishes at the boundary.

${ }^{10}$ The $A d S_{4}$ metric is $\mathrm{d} s^{2}=\frac{1}{r^{2}} \eta_{\mu \nu} \mathrm{d} x^{\mu} \mathrm{d} x^{\nu}$, where $\eta_{\mu \nu}$ is the Minkwoski metric, which we use to raise, lower and contract four-vector indices.
} 
and van der Waerden symbols ${ }^{11}$, the different objects entering this expression can be defined as

$$
\begin{array}{r}
x_{0, i}^{\mu}:=x_{0}^{\mu}-x_{i}^{\mu}, \quad K_{i}:=\frac{r_{0}}{\left(x_{0, i}\right)^{2}}, \quad \Sigma_{i}:=\sigma^{r}-\frac{2 r_{0}}{\left(x_{0, i}\right)^{2}} x_{0, i}, \\
v_{i}:=\frac{\sqrt{2 r_{0}}}{\left(x_{0, i}\right)^{2}} \Sigma_{i} \bar{x}_{0, i} \chi_{i}, \quad\left(v_{i}\right)^{\dagger}=\bar{v}_{i}=-\bar{\Sigma}_{i} v_{i} .
\end{array}
$$

In the parity-invariant models, the resulting pre-amplitudes read

$$
\begin{aligned}
\mathscr{A}_{n_{0}, t}^{\mathrm{A}-\text { model }}\left(\mathscr{K}_{i}\right) & =\beta_{n_{0}, t} \exp \left(-\frac{i}{4} \sum_{i=1}^{n_{0}} Q_{i}\right)\left(\prod_{i=1}^{n_{0}} \frac{\cos \left(\frac{1}{2} P_{i, i+1}\right)}{\left|x_{i, i+1}\right|}\right), \\
\mathscr{A}_{n_{0}, t}^{\mathrm{B}-\operatorname{model}}\left(\mathscr{K}_{i}\right) & =-(-1)^{t} \beta_{n_{0}, t} \exp \left(-\frac{i}{4} \sum_{i=1}^{n_{0}} Q_{i}\right)\left(\prod_{i=1}^{n_{0}} \frac{\sin \left(\frac{1}{2} P_{i, i+1}\right)}{\left|x_{i, i+1}\right|}\right), \\
\beta_{n_{0}, t}: & =4(i)^{2 n_{0}-2+e+t}(2 \pi)^{2+e+t} \prod_{j=1}^{n_{0}} \operatorname{sgn}\left(x_{j, j+1}^{2}\right),
\end{aligned}
$$

where $\operatorname{sgn}(x)$ is the sign function, $x_{i, j}:=x_{i}-x_{j},|x|:=\sqrt{x^{2}}$ and where

$$
\begin{aligned}
P_{i, i+1} & :=\frac{1}{\left(x_{i, i+1}\right)^{2}} \chi_{i} \sigma^{r} \bar{x}_{i, i+1} \chi_{i+1}, \\
Q_{i} & :=\chi_{i} \sigma^{r}\left(\frac{\bar{x}_{i, i+1}}{\left(x_{i, i+1}\right)^{2}}-\frac{\bar{x}_{i, i-1}}{\left(x_{i, i-1}\right)^{2}}\right) \chi_{i},
\end{aligned}
$$

are real conformally invariant variables [24].

Once bose-symmetrized, this expression reproduces, up to constant coefficients, the result obtained by combining the equations (6.19) and (6.20) of [25] and generalises it to the case $t=1$, where the only difference at this order is a global sign in the Type B model.

Turning to the free $U(N)$ vector model in $d$-dimensions, it is constructed from a complex Lorentz scalar $\left(\phi^{i}, \phi_{i}^{*}\right)$, where $i=1, \ldots, N$, with two-point functions

$$
\left\langle\phi^{i}(x) \phi^{j}(y)\right\rangle=0=\left\langle\phi_{i}^{*}(x) \phi_{j}^{*}(y)\right\rangle, \quad\left\langle\phi^{i}(x) \phi_{j}^{*}(y)\right\rangle=\frac{\delta_{j}^{i}}{|x-y|^{d-2}} .
$$

The conserved current $J_{\mu(s)}$ of spin $s$ is a bilinear operator that is a $U(N)$ singlet traceless tensor containing $s$ spacetime-derivatives. Using a polarisation vector $\varepsilon^{\mu}$ and some weights $a_{s}$, one can gather all the conserved currents into a generating function ${ }^{12}$ :

$$
J(x, \varepsilon)=\sum_{s=0}^{\infty} a_{s} J_{\mu(s)}(x)\left(\varepsilon^{\mu}\right)^{s}=\phi_{i}^{*}(x) f(\varepsilon, \overleftarrow{\partial}, \vec{\partial}) \phi^{i}(x)
$$

The function $f$ is determined by the condition that $J_{\mu(s)}$ be conserved on-shell, Lorentz-covariant and traceless. After requiring $\varepsilon$ to be null ${ }^{13}$ and choosing a normalization of the currents, it can be written

$$
f(\varepsilon, u, v)=\sum_{s, k}\left(\frac{i}{4}\right)^{s} \frac{\Gamma\left(\frac{d-2}{2}\right)}{k ! s ! \Gamma\left(k+\frac{d-2}{2}\right) \Gamma\left(s-k+\frac{d-2}{2}\right)}(-\varepsilon \cdot u)^{k}(\varepsilon \cdot v)^{s-k} .
$$

\footnotetext{
${ }^{11} \mathrm{~A}$ vector $v^{\mu}$ is implicitly associated with two matrices $v_{\alpha \dot{\beta}}:=v^{\mu} \sigma_{\mu \alpha \dot{\beta}}$ and $\bar{v}^{\dot{\alpha} \beta}:=v^{\mu} \bar{\sigma}_{\mu}{ }^{\dot{\alpha} \beta}$.

${ }^{12}$ See e.g. [35, 11, 36] and references therein.

${ }^{13}$ This is done in order to eliminate the ambiguity caused by the possibility of making trivial transformations of the form $\left(\varepsilon^{\mu}\right)^{s} \rightarrow\left(\varepsilon^{\mu}\right)^{s}+\left(\eta^{\mu(2)}\right)^{\ell}\left(\varepsilon^{2}\right)^{\ell}\left(\varepsilon^{\mu}\right)^{s-2 \ell}$.
} 
The connected correlation functions $\left\langle J_{1} \cdots J_{n_{0}}\right\rangle_{\text {conn. }}$ are given in terms of cyclically-symmetric building blocks

$$
\left\langle J_{1}, \ldots, J_{n_{0}}\right\rangle_{\mathrm{cyclic}}:=\left.\frac{1}{N} \prod_{i=1}^{n_{0}} f\left(\varepsilon_{i}, \partial_{x_{i}^{\prime}}, \partial_{x_{i}}\right) \prod_{j=1}^{n_{0}}\left\langle\phi^{i_{j}}\left(x_{j}\right) \phi_{i_{j+1}^{*}}^{*}\left(x_{j+1}^{\prime}\right)\right\rangle\right|_{x_{k}^{\prime}=x_{k} \forall k, x_{n_{0}+1}=x_{1}} .
$$

After some algebraic manipulations, they can be given the form

$$
\left\langle J_{1}, \ldots, J_{n_{0}}\right\rangle_{\text {cyclic }}=\prod_{i=1}^{n_{0}} \exp \left(-\frac{i}{4} Q_{i}\right) \sum_{c_{i}} \frac{1}{c_{i} ! \Gamma\left(c_{i}+\frac{d-2}{2}\right)}\left(\frac{i}{4} P_{i, i+1}\right)^{2 c_{i}}\left|x_{i, i+1}\right|^{2-d},
$$

where

$$
\begin{aligned}
Q_{i} & :=2 \varepsilon_{i} \cdot\left(\frac{x_{i-1, i}}{\left(x_{i-1, i}\right)^{2}}+\frac{x_{i, i+1}}{\left(x_{i, i+1}\right)^{2}}\right), \\
P_{i, i+1}^{2} & :=\frac{4}{\left(x_{i, i+1}\right)^{4}}\left(\left(\varepsilon_{i} \cdot x_{i, i+1}\right)\left(\varepsilon_{i+1} \cdot x_{i, i+1}\right)-\frac{1}{2}\left(\varepsilon_{i} \cdot \varepsilon_{i+1}\right)\left(x_{i, i+1}\right)^{2}\right),
\end{aligned}
$$

are (real) conformally invariant variables. This result confirms the conjecture of [37].

In the case of the three-dimensional $U(N)$ model, the result can further be rewritten as

$$
\left\langle J_{1}, \ldots, J_{n_{0}}\right\rangle_{\text {cyclic }}=\prod_{i=1}^{n_{0}} \frac{1}{\sqrt{\pi}} \exp \left(-\frac{1}{4} Q_{i}\right) \cos \left(\frac{1}{2} P_{i, i+1}\right)\left|x_{i, i+1}\right|^{2-d} .
$$

Upon identifying

$$
\varepsilon_{i}=-\frac{1}{2} \sigma^{\alpha \dot{\alpha}}\left(\chi_{i}\right)_{\alpha}\left(\bar{\chi}_{i}\right)_{\dot{\alpha}}
$$

the conformally invariant variables agree, and one has

$$
\left\langle J_{1}, \ldots, J_{n_{0}}\right\rangle_{\text {cyclic }}=\mathscr{A}_{n_{0}, t}^{\mathrm{A}-\text { model }}\left(\mathscr{K}_{i}\right)
$$

at the level of cyclic structures, thus strengthening the previously obtained correspondence [25, 26], which was obtained at the level of completely symmetric structures. We also note that after bosesymmetrization, the form of the correlation functions agrees with that obtained in [27].

The cyclic structures of the free $O(N)$ vector model can be obtained from those of $U(N)$ ditto by projecting onto the part that is even with respect to all of the $\varepsilon_{i}$. Indeed, in the $O(N)$ model, the free field two-point functions

$$
\left\langle\phi^{i}(x) \phi^{j}(y)\right\rangle=\frac{\delta^{i j}}{|x-y|^{d-2}} .
$$

Thus, while there are two ways to contract two currents in the $U(N)$ model, there are four ways to perform this operation in the $O(N)$ model, which leads to the aforementioned symmetrization of the dependence on the polarization vectors. On the bulk side, this operation is equivalent to imposing the minimal bosonic projection condition on the Weyl zero-form, viz.

$$
\begin{aligned}
\tilde{\mathscr{K}}_{i}^{M B}\left(x_{0}, x_{i}, \chi_{i} \mid \Lambda_{i}\right): & =\frac{1}{2}(1+\pi \tau) \mathscr{K}_{i}\left(x_{0}, x_{i}, \chi_{i} \mid Y\right) \\
& =\frac{1}{2}\left(\tilde{\mathscr{K}_{i}}\left(x_{0}, x_{i}, \chi_{i} \mid Y\right)+\tilde{\mathscr{K}_{i}}\left(x_{0}, x_{i}, i \chi_{i} \mid Y\right)\right),
\end{aligned}
$$

which is indeed equivalent to symmetrization on $\varepsilon_{i}$, which is bilinear in $\chi_{i}$, thereby confirming the statement. 


\section{Conclusions and Outlook}

We have shown that instead of seeking an effective formulation of Vasiliev theory on $\mathscr{X}_{4}$, studying the full theory on $\mathscr{X}_{4} \times \mathscr{Z}_{4}$ is interesting on its own. Indeed the parallel with noncommutative Yang-Mills theories allows to get results sensible to holography through the evaluation of Wilson lines that are open along the noncommutative directions. In order to push further this analogy, it would be interesting to start by looking at the three-dimensional version of Vasiliev's model [38] which is known [39] to admit a noncommutative Yang-Mills action in Z-space.

We also have shown that the evaluation at the leading order of the Wilson lines in type A model on $n$ boundary-to-bulk propagators (with the scalar one obeying Neumann b.c.) connecting $n$ separated points on the boundary to a given base point in the bulk, reproduces corresponding correlators of conserved currents in the free $U(N)$ vector model. However, since both bulk fields and gauge transformations receive corrections in classical perturbation theory, the leading order is not the end of the story, and all orders need to be included into the Wilson lines for these to capture Vasiliev's theory more fully. The door is still open for non-trivial subleading corrections that could correspond to contact terms on the CFT-side, whereby in particular Vasiliev's type A model in $A d S_{4}$ would be a dual of a genuinely interacting bosonic $C F T_{3}$.

This setting can in principle be used to probe other dualities than the simplest $A d S_{4} / C F T_{3}$ one. It would be interesting to study the perturbative expansions around asymptotically $A d S_{4}$ backgrounds, where possible contact terms become active as part of the boundary field theory Lagrangian. One may also push the results on the other boundary condition [26] beyond the leading order and adapt the method to the parity breaking models. Eventually, the new result on $n$-point functions on the $C F T_{d}$ makes possible to repeat the bulk analysis in Vasiliev's $(d+1)$-dimensional type A model [40] where all the ingredients can be reconstructed.

\section{Acknowledgements}

D.D.F. wants to thank the organisers of the Corfu Summer Institute 2017 "School and Workshops on Elementary Particle Physics and Gravity", and in particular the ones of the Training School "Quantum Spacetime and Physics Models", where this work was presented. The work of R.B. is supported by a PDR "Gravity and extensions" from the F.R.S.-FNRS (Belgium). N.B. is F.R.S.-FNRS Senior Research Associate (Belgium). D.D.F is F.R.S.-FNRS Research Fellow. The work of P.S. is supported by Fondecyt Regular grant No 1140296, Conicyt grant DPI 20140115 and UNAB internal grant DI-1382-16/R.

\section{References}

[1] X. Bekaert, N. Boulanger and P. A. Sundell, How higher-spin gravity surpasses the spin-two barrier, Rev. Mod. Phys. 84 (2012) 987 [1007.0435].

[2] M. A. Vasiliev, Consistent equation for interacting gauge fields of all spins in (3+1)-dimensions, Phys. Lett. B243 (1990) 378.

[3] C. Fronsdal, Massless Fields with Integer Spin, Phys. Rev. D18 (1978) 3624. 
[4] E. Sezgin and P. Sundell, Massless higher spins and holography, Nucl. Phys. B644 (2002) 303 [hep-th/0205131].

[5] I. R. Klebanov and A. M. Polyakov, AdS dual of the critical $O(N)$ vector model, Phys. Lett. B550 (2002) 213 [hep-th/0210114].

[6] R. G. Leigh and A. C. Petkou, Holography of the $N=1$ higher-spin theory on AdS(4), JHEP 06 (2003) 011 [hep-th/0304217].

[7] E. Sezgin and P. Sundell, Holography in 4D (super) higher spin theories and a test via cubic scalar couplings, JHEP 0507 (2005) 044 [hep-th/ 030504 0].

[8] S. Giombi, S. Minwalla, S. Prakash, S. P. Trivedi, S. R. Wadia et al., Chern-Simons Theory with Vector Fermion Matter, Eur.Phys.J. C72 (2012) 2112 [1110.4386].

[9] O. Aharony, G. Gur-Ari and R. Yacoby, Correlation Functions of Large N Chern-Simons-Matter Theories and Bosonization in Three Dimensions, JHEP 1212 (2012) 028 [1207. 4 593].

[10] R. Bonezzi, N. Boulanger, D. De Filippi and P. Sundell, Noncommutative Wilson lines in higher-spin theory and correlation functions of conserved currents for free conformal fields, J. Phys. A50 (2017) $475401[1705.03928]$.

[11] S. Giombi and X. Yin, Higher Spin Gauge Theory and Holography: The Three-Point Functions, JHEP 09 (2009) 115 [0912.3462].

[12] S. Giombi and X. Yin, Higher Spins in AdS and Twistorial Holography, JHEP 04 (2010) 086 [1004.3736].

[13] N. Boulanger, P. Kessel, E. D. Skvortsov and M. Taronna, Higher spin interactions in four-dimensions: Vasiliev versus Fronsdal, J. Phys. A49 (2016) 095402 [1508. 04139 ].

[14] C. Sleight and M. Taronna, Higher spin gauge theories and bulk locality: a no-go result, 1704.07859 .

[15] M. A. Vasiliev, On the Local Frame in Nonlinear Higher-Spin Equations, JHEP 01 (2018) 062 [1707.03735].

[16] D. Ponomarev, A Note on (Non)-Locality in Holographic Higher Spin Theories, Universe 4 (2017) 2 [1710.00403].

[17] N. Boulanger, S. Leclercq and P. Sundell, On The Uniqueness of Minimal Coupling in Higher-Spin Gauge Theory, JHEP 0808 (2008) 056 [0805 . 2764].

[18] N. Colombo and P. Sundell, Twistor space observables and quasi-amplitudes in 4D higher spin gravity, JHEP 1111 (2011) 042 [1012.0813].

[19] J. Engquist and P. Sundell, Brane partons and singleton strings, Nucl. Phys. B752 (2006) 206 [hep-th/0508124].

[20] E. Sezgin and P. Sundell, An exact solution of 4D higher-spin gauge theory, Nucl. Phys. B762 (2007) 1 [hep-th/0508158].

[21] C. Iazeolla and P. Sundell, Families of exact solutions to Vasiliev's 4D equations with spherical, cylindrical and biaxial symmetry, JHEP 1112 (2011) 084 [1107.1217].

[22] C. Iazeolla and P. Sundell, Biaxially symmetric solutions to 4D higher-spin gravity, J.Phys. A46 (2013) 214004 [1208.4077]. 
[23] C. Iazeolla and P. Sundell, 4D Higher Spin Black Holes with Nonlinear Scalar Fluctuations, JHEP 10 (2017) 130 [1705.06713].

[24] N. Colombo and P. Sundell, Higher Spin Gravity Amplitudes From Zero-form Charges, 1208 . 3880.

[25] V. Didenko and E. Skvortsov, Exact higher-spin symmetry in CFT: all correlators in unbroken Vasiliev theory, JHEP 1304 (2013) 158 [1210 . 7963].

[26] V. Didenko, J. Mei and E. Skvortsov, Exact higher-spin symmetry in CFT: free fermion correlators from Vasiliev Theory, Phys. Rev. D88 (2013) 046011 [1301.4166].

[27] O. A. Gelfond and M. A. Vasiliev, Operator algebra of free conformal currents via twistors, Nucl. Phys. B876 (2013) 871 [1301.3123].

[28] M. A. Vasiliev, Higher spin algebras and quantization on the sphere and hyperboloid, Int.J.Mod.Phys. A6 (1991) 1115.

[29] E. P. Wigner, Do the Equations of Motion Determine the Quantum Mechanical Commutation Relations?, Phys. Rev. 77 (1950) 711.

[30] L. M. Yang, A Note on the Quantum Rule of the Harmonic Oscillator, Phys. Rev. 84 (1951) 788.

[31] D. J. Gross, A. Hashimoto and N. Itzhaki, Observables of noncommutative gauge theories, Adv. Theor. Math. Phys. 4 (2000) 893 [hep-th/ 0008075 ].

[32] J. Ambjorn, Y. Makeenko, J. Nishimura and R. Szabo, Lattice gauge fields and discrete noncommutative Yang-Mills theory, JHEP 0005 (2000) 023 [hep-th/ 0004147 ].

[33] Yu. Makeenko, Methods of contemporary gauge theory. Cambridge University Press, 2005.

[34] E. Sezgin and P. Sundell, Geometry and Observables in Vasiliev's Higher Spin Gravity, JHEP 07 (2012) 121 [1103.2360].

[35] N. S. Craigie, V. K. Dobrev and I. T. Todorov, Conformally Covariant Composite Operators in Quantum Chromodynamics, Annals Phys. 159 (1985) 411.

[36] S. Giombi, Higher Spin âĂ ̌̌ CFT Duality, in Proceedings, Theoretical Advanced Study Institute in Elementary Particle Physics: New Frontiers in Fields and Strings (TASI 2015): Boulder, CO, USA, June 1-26, 2015, pp. 137-214, 2017, 1607.02967 , DOI.

[37] C. Sleight and M. Taronna, Higher Spin Interactions from Conformal Field Theory: The Complete Cubic Couplings, Phys. Rev. Lett. 116 (2016) 181602 [1603.00022].

[38] S. F. Prokushkin and M. A. Vasiliev, Higher-spin gauge interactions for massive matter fields in 3D AdS space-time, Nucl. Phys. B545 (1999) 385 [hep-th/9806236].

[39] S. F. Prokushkin, A. Y. Segal and M. A. Vasiliev, Coordinate free action for AdS(3) higher spin matter systems, Phys.Lett. B478 (2000) 333 [hep-th/9912280].

[40] M. A. Vasiliev, Nonlinear equations for symmetric massless higher spin fields in (A)dS(d), Phys. Lett. B567 (2003) 139 [hep-th/0304049]. 